\title{
Tellus: um modelo computacional para a predição da fertilidade do solo na agricultura de precisão
}

\author{
Gilson A. Helfer ${ }^{1,2}$, Bruno G. Martini ${ }^{1}$, Ronaldo B. dos Santos ${ }^{3}$, \\ Adilson Ben da Costa ${ }^{3}$, Jorge L. V. Barbosa ${ }^{1}$ \\ ${ }^{1}$ Programa de Pós-Graduação em Computação Aplicada (PPGCA) \\ Universidade do Vale do Rio dos Sinos (UNISINOS) \\ Av. Unisinos, 950 - 93.022-750 - São Leopoldo - RS - Brazil \\ ${ }^{2}$ Departamento de Computação - Universidade de Santa Cruz do Sul (UNISC) \\ ${ }^{3}$ Programa de Pós-Graduação em Sistemas e Processos Industrais (PPGSPI) \\ Universidade de Santa Cruz do Sul (UNISC)

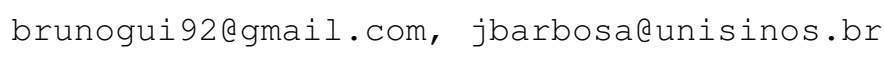

\begin{abstract}
The application of ubiquitous computing has increased in recent years, especially due to the development of technologies such as mobile computing and its integration with the real world. One of the challenges in this area is the use of context awareness. In agriculture, the context can be related to the environment, for example, the chemical and physical aspects that characterize different types of soil over time. This paper proposes a computational model applied in precision agriculture that uses the contexts history to predict soil fertility. The best results were obtained in the prediction of organic matter, with a coefficient of determination $\left(R^{2}\right)$ of 0.9102 for root mean square error (RMSE) of $0.49 \%$.
\end{abstract}

Resumo. A aplicação da computação ubíqua tem aumentado nos últimos anos, especialmente devido ao desenvolvimento de tecnologias como a computação móvel e sua integração com o mundo real. Um dos desafios nessa área é o uso da sensibilidade ao contexto. Na agricultura, pode-se considerar o contexto relacionado ao meio ambiente, por exemplo, os aspectos químicos e físicos que caracterizam os diferentes tipos de solo ao longo do tempo. Este artigo propõe um modelo computacional aplicado na agricultura de precisão que usa os históricos de contextos para predição da fertilidade do solo. Os melhores resultados foram obtidos na predição de matéria orgânica, com um coeficiente de determinação $\left(R^{2}\right)$ de 0.9102 para um erro quadrático médio (RMSE) de $0.49 \%$.

\section{Introdução}

A aplicação da agricultura de precisão tem se mostrado uma estratégia adequada para aumentar a produtividade, permitindo o uso racional de insumos e reduzindo os impactos ambientais causados pelas práticas agrícolas. Atualmente, os insumos são utilizados de forma variável, visando atender as necessidades específicas de cada localidade, otimizando o processo produtivo. Entretanto, é necessário caracterizar a variabilidade dos 
atributos químicos e físicos do solo por meio de uma amostragem representativa de tais variações [Costa et al. 2014].

A análise de solos é o único método que permite, antes do plantio, conhecer a capacidade de um determinado solo suprir nutrientes para as plantas. É a forma mais simples, econômica e eficiente de diagnose da fertilidade das terras e constitui base relevante para a recomendação de quantidades adequadas de corretivos e fertilizantes para aumentar a produtividade das culturas e, como consequência, a produção e a lucratividade das lavouras. Programas de controle de qualidade inter-laboratoriais como a Rede Oficial de Laboratórios de Análise de Solo e de Tecido Vegetal dos Estados do Rio Grande do Sul e de Santa Catarina (ROLAS) garantem o monitoramento dos erros de medição das análises de solos e, consequentemente, a minimização de erros nas recomendações de adubação e calagem, além de sugerirem métodos de análises [Griebeler et al. 2016].

Muitas das análises sugeridas pela ROLAS, ditas como oficiais, são caras, geram resíduos poluentes além de um enorme tempo de preparação das amostras. A análise de matéria orgânica e argila, que de uma forma geral representam a fertilidade do solo em questão, demandam 21 horas para determinação de seus valores. Além disso, a primeira citada, gera resíduos extremamente danosos ao meio ambiente. Em contrapartida, técnicas modernas vêm sendo estudadas nos últimos anos para substituírem as técnicas oficiais. Dentre elas pode-se citar a espectroscopia molecular na região do infravermelho próximo (NIR) [Muñoz and Kravchenko 2011].

A quimiometria permite relacionar as medidas realizadas em um método analítico (espectroscopia molecular, por exemplo), obtendo informações através da aplicação de métodos matemáticos e estatísticos, permitindo a manipulação de grandes quantidades de dados. Ela pode ser dividida em três áreas principais: planejamento e otimização de experimentos, reconhecimento de padrões e calibração multivariada. Nesse sentido, várias ferramentas de Machine Learning vêm sendo aplicadas na quimiometria para calibração multivariada. Esses algoritmos eliminam variáveis que não se correlacionam com a propriedade de interesse, como aquelas que adicionam ruído, não-linearidades ou informações irrelevantes [Ferreira 2015].

Uma vez selecionadas as variáveis, métodos como Mínimos Quadrados Parciais (Partial Least Squares) e Regressão Linear Múltipla (Multiple Linear Regression) geralmente são aplicados, principalmente em situações onde variáveis de processo apresentam elevados níveis de correlação, ruído, observações faltantes e desbalanço na proporção de variáveis e observações. Na regressão é gerada um reduzido número de combinações lineares independentes das variáveis de processo. Essas novas variáveis, chamadas de componentes, respondem pela maior parte da variância presente nas variáveis originais do processo. Normalmente, apenas três ou quatro componentes são retidos para representar dezenas ou mesmo centenas de variáveis de processo [Anzanello 2013].

Para a computação ubíqua, um modelo computacional baseado em um framework e um middleware são comumente usados. Nele, encontram-se métodos utilizados para comunicação remota, tolerância à falhas, alta disponibilidade, acesso à informação remota e segurança, entre outros. Um aspecto fundamental para que essa visão se torne realidade é a possibilidade de desenvolver aplicações que sejam sensíveis ao contexto [Dey et al. 2001]. Através do conhecimento de dados contextuais, uma aplicação pode 
ajustar seu próprio funcionamento ou até mesmo agir de forma proativa, alertando o usuário para um cenário específico ou auxiliando-o a desenvolver suas atividades com mais eficiência. As informações geradas permitirão a construção de um banco de dados histórico para posterior tomada de decisão [Rosa et al. 2015], [Barbosa et al. 2018].

No presente artigo é proposto um modelo computacional denominado Tellus, uma arquitetura que utiliza o paradigma de computação ubíqua na agricultura de precisão para predição da fertilidade do solo através de histórico de contextos. O artigo está organizado da seguinte forma: a seção 2 cita e descreve alguns trabalhos relacionados; a seção 3 apresenta o modelo proposto e a implementação da arquitetura; a seção 4 mostra o resultado de alguns experimentos e análises quantitativas; e, finalmente, na seção 5 é apresentada a conclusão.

\section{Trabalhos relacionados}

Foram considerados como trabalhos relacionados apenas as pesquisas que propuseram realizar predições de dados para tomada de decisão na agricultura de precisão. O comparativo entre os trabalhos, incluindo o Tellus, é apresentado na Tabela 1.

[Nawar and Mouazen 2019] demonstraram o potencial de usar um espectrômetro on-the-go para realizar medições em tempo real por meio de espectroscopia de refletância de infravermelho próximo. Foram gerados modelos de calibração para matéria orgânica visando comparar resultados online (acoplado a um trator) e resultados de laboratório. Desempenho de previsão menos preciso foi obtido para a predição on-line em comparação com a predição de laboratório.

[Huong et al. 2018] propuseram um modelo genérico utilizando Decisão de Markov para criar uma irrigação automática e precisa e que torne a agricultura mais eficiente em consumo de energia e água. [Treboux and Genoud 2018] apresentaram o impacto do aprendizado de máquina junto a agricultura de precisão na segregação de cores em imagens de satélites. [Goap et al. 2018] propuseram um sistema inteligente programado em código aberto para prever os requisitos de irrigação do campo com utilização de vários sensores de medições físicas do solo e ambientais.

[Santos et al. 2018] apresentaram um modelo de arquitetura de redes de sensores sem fio para realizar predição de umidade e temperatura do solo. Os resultados obtidos sinalizaram a viabilidade da proposta e limitações quanto ao acompanhamento em tempo real do cultivo e nos mecanismos de segurança para a transmissão dos dados. Já [Concepcion et al. 2014] projetaram e implementaram uma rede de sensores sem fio para para permitir a coleta de dados do ambiente agrícola, como temperatura, umidade e luz.

Para realizar a análise comparativa foram estabelecidos critérios como o tipo de sensores empregados, a proposta de uma arquitetura, o método aplicado para a análise dos dados e local do experimento. Uma vez analisados os trabalhos relacionados, não foram encontrados na literatura trabalhos diretamente relacionados à predição de fertilidade de solos na agricultura de precisão baseados na análise de históricos de contextos. As pesquisas que propuseram um modelo computacional e dados de sensores para tomada de decisão, fizeram na sua maioria uso de sensores de umidade e temperatura, ou seja, apenas aspectos físicos do solo ou do ambiente, e não químicos/orgânicos, como sugere este artigo. 
Tabela 1. Comparativo dos trabalhos relacionados

\begin{tabular}{|l|c|c|c|r|}
\hline Artigo & Arquitetura & Sensores & Análise & Local \\
\hline [Nawar and Mouazen 2019] & Não & Infravermelho & Mach. Learning & Bélgica \\
\hline [Huong et al. 2018] & Não & Umidade & Decisão Markov & Vietnã \\
\hline [Treboux and Genoud 2018] & Não & Imagens de satélite & Mach. Learning & Suiça \\
\hline [Goap et al. 2018] & Sim & UV, Temp. e Umidade & Mach. Learning & Índia \\
\hline [Santos et al. 2018] & Sim & Temperatura e Umidade & Arima & Brasil \\
\hline [Concepcion et al. 2014] & Sim & Câmera, Temp. e Umid. & N/A & Itália \\
\hline Tellus & Sim & Infravermelho & Mach. Learning & Brasil \\
\hline
\end{tabular}

\section{Modelo Tellus}

O modelo Tellus tem a finalidade de predizer dados referentes a fertilidade do solos a partir de uma base histórica. Para isso, o modelo utiliza dados de sensores de infravermelho próximo, o período e a localização que a análise foi realizada. Todas estas informações são armazenadas para futuras predições. Frente à inexistência de um modelo computacional que realize estas análises de uma maneira rápida, não invasiva e sem geração de resíduos poluentes, busca-se responder a questão principal que fundamenta o trabalho: "Como seria uma arquitetura que utilizasse dados de sensores onipresentes para predição de matéria orgânica e argila para agricultura de precisão?’”.

\subsection{Arquitetura e histórico de contextos}

O modelo Tellus foi projetado usando a modelagem técnica de arquitetura da SAP (TAM, do inglês, Standard for Technical Architecture Modeling) [SAP AG 2007]. Na Figura 1 é apresentada a arquitetura composta por atores (A1, A2 e A3), acessos, blocos (Assistente Móvel, Atuador e Servidor) e componentes. Os componentes aparecem na parte interna dos blocos, também são apresentados os canais de comunicação, indicados pelos símbolos $\mathrm{Cl}, \mathrm{C} 2$ e $\mathrm{C} 3$.

O Tellus organiza o fluxo das informações de forma a obter dados gerados pelos atores, e após tratados, disponibiliza informações contextuais de acordo com a requisição realizada. Para isso o modelo contempla três blocos: um assistente móvel, um middleware para sensores conectados em atuadores e um servidor.

O assistente móvel, recebe as ações do cliente móvel (A1) por meio do canal de comunicação $\mathrm{C} 1$ e realiza comunicação com o servidor utilizando webservices a partir de métodos RESTful a fim de demonstrar um mapa em tempo real nas condições de fertilidade do solo baseados em georreferenciamento.

O atuador agrícola, constituído de um middleware, é responsável por atender os casos de uso do cliente sensor (A2), pelo canal C2, possuindo métodos de comunicação para envio de dados ao servidor e também para recebimento de atualizações e novas configurações. O agente atuador coleta os dados do solo e também notifica se o local onde o tratorista se encontra é crítico para tratamento. Esta notificação é realizada após a predição de matéria orgânica e argila e é disparada para o assistente móvel. Com base nessas análises, ele verifica se há necessidade de correção de solo com fertilizantes além da quantidade a ser utilizada. Este agente insere marcações nos históricos de con- 


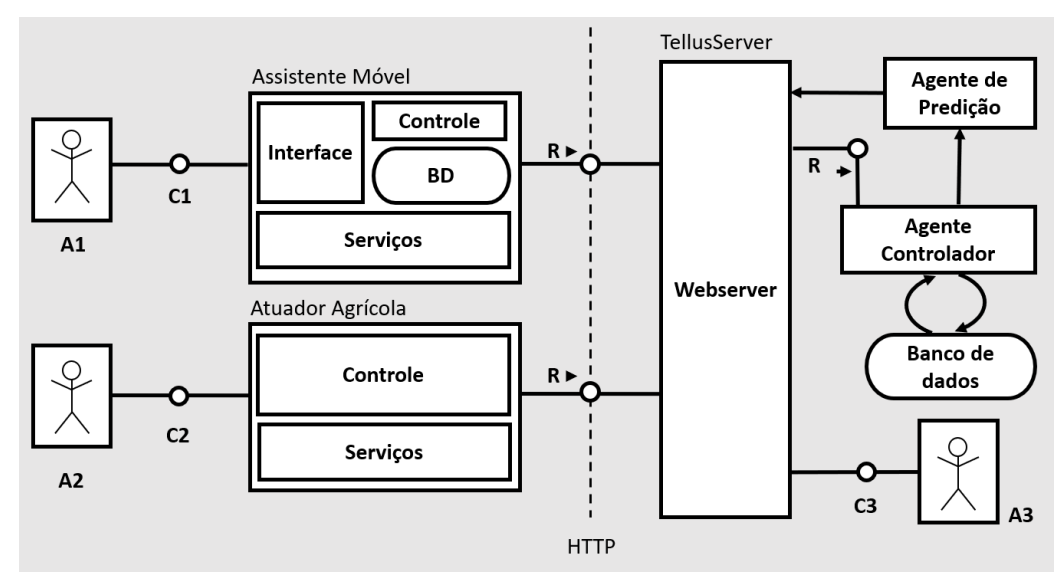

Figura 1. Arquitetura do modelo Tellus.

texto correspondentes. Para gerenciamento dos históricos de contexto são armazenadas as informações apresentadas na Tabela 2.

Tabela 2. Propriedade dos históricos de contexto

\begin{tabular}{|l|c|c|}
\hline Propriedade & Formato & Descrição \\
\hline 1. Evento & Timestamp & Armazena data e hora corrente \\
\hline 2. Latitude & Decimal & Latitude no formato decimal 17 dígitos \\
\hline 3. Longitude & Decimal & Longitude no formato decimal 17 dígitos \\
\hline 4. Infravermelho & String & Dados espectrais no formato CSV \\
\hline 5. Matéria orgânica & Decimal & Concentração de matéria orgânica \\
\hline 6. Argila & Decimal & Concentração de argila \\
\hline 7. IP & String & Armazena o endereço IP da requisição \\
\hline
\end{tabular}

O componente Servidor, intitulado TellusServer, realiza comunicação com os demais componentes por canais internos e com o ator dados externos (A3), pelo canal C3. O Servidor contempla mecanismos para tratamento e predição dos dados referente às análises de solo (dados espectrais) e localização. A partir daí, utilizando algoritmos de regressão linear múltipla, é realizada a predição dos resultados de matéria orgânica e argila, vinculados aos históricos da Tabela 2.

\subsection{Representação das entidades}

Uma ontologia foi proposta para padronização das informações visando sua interoperabilidade e preservação. Dentre as utilizadas na agricultura, destaca-se o AgroRDF, baseado no padrão AgroXML [Blank et al. 2013], que vem sendo desenvolvido desde 2004 para comunicação de dados entre agricultores e outros parceiros da cadeia de suprimentos [Schmitz et al. 2009]. Para representar os ambientes, resultados, recursos e condições da amostra foi realizada uma extensão do AgroRDF, conforme mostra a Figura 2.

Foram adicionadas as subclasses IP, Latitude e Longitude na classe Point, Infrared na SoilSample, OM (Organic Matter) e Clay na classe SoilAnalysis. 


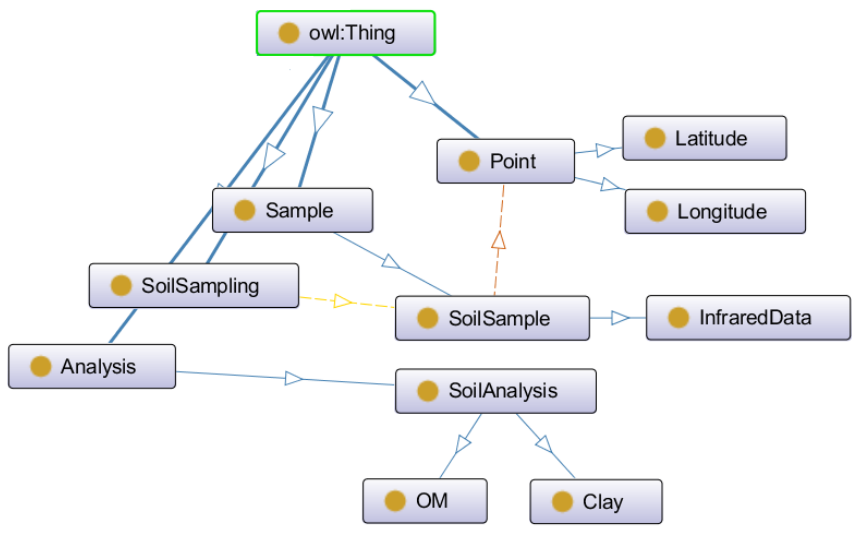

Figura 2. Detalhe AgroRDF proposto

\subsection{Implementação}

O assistente móvel foi implementado para os dispositivos com sistema operacional Android. Dessa forma, utilizando a IDE Android Studio, foram desenvolvidas duas interfaces: uma tela de login (A) e uma tela onde fosse possível visualizar os pontos de coletas de infravermelho com a respectiva predição dos resultados de matéria orgânica e argila (B), conforme ilustra a Figura 3.
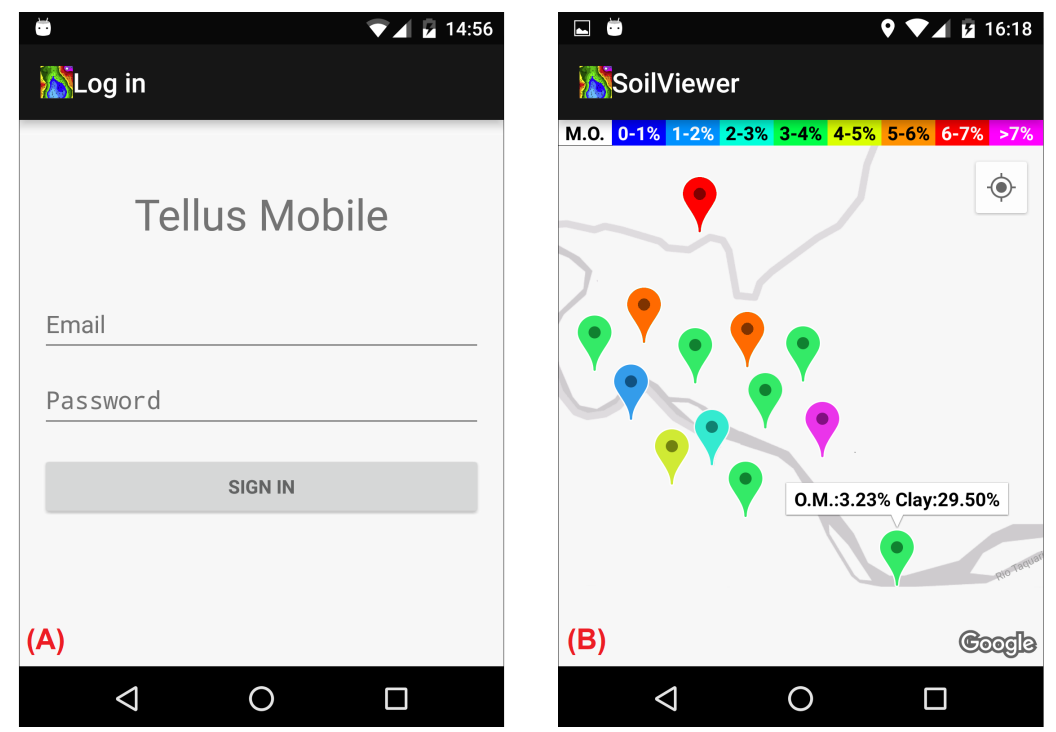

Figura 3. Interface do aplicativo: tela de login (A) e tela de visualização (B)

No lado do servidor o agente de predição foi implementado em linguagem Python utilizando a biblioteca de Machine Learning Scikit-Learn. O atuador agrícola consiste em um sensor móvel de infravermelho próximo (Texas Instruments DLP NIRScan Nano) acoplado em um Raspberry Pi Model 3. São também conectados um módulo GPS (GYGPS6MV2) para capturar a latitude e longitude do local e um módulo GPRS (SIM800L) para comunicação dos dados. Um exemplo de base de dados de históricos de contextos referente ao modelo Tellus é ilustrado na Figura 4.

Foi selecionado um conjunto de 355 amostras de solos de diversos pontos de 


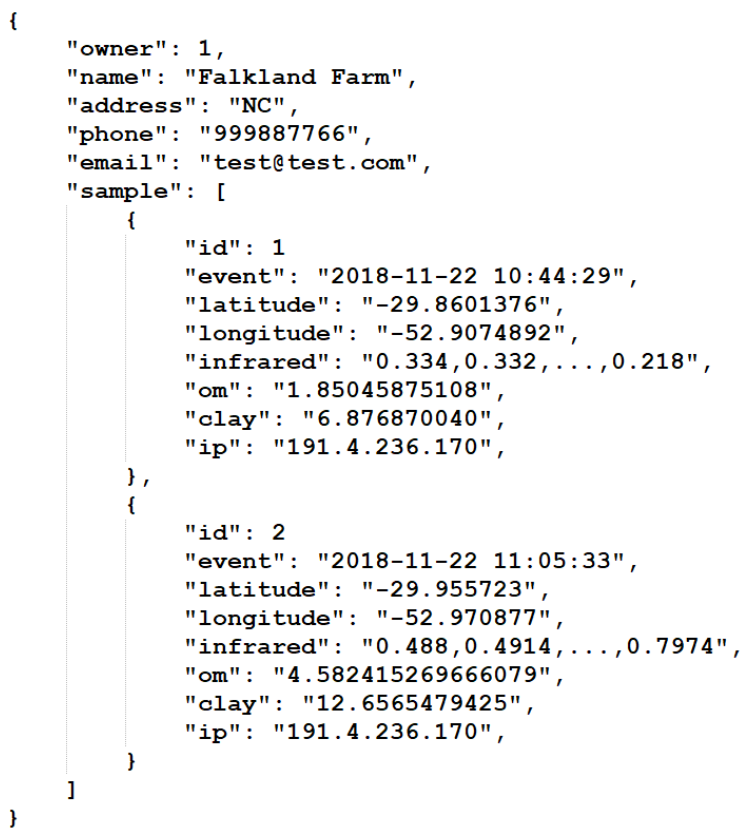

Figura 4. Exemplo do histórico de contextos do modelo Tellus

coleta do Vale do Rio Pardo, cujas concentrações de matéria orgânica variaram entre $0.41 \%$ e $7.42 \%$ e argila entre $4 \%$ e $72 \%$. Essas amostras foram fornecidas pelo setor Central Analítica (UNISC), onde realizaram a secagem em estufa modelo Marconi-MA037 com circulação de ar por um período de no mínimo 24 horas com temperatura entre 45 e $60^{\circ} \mathrm{C}$. Após, as amostras foram moídas em moinho de martelo modelo MarconiN1040, com peneira de $2 \mathrm{~mm}$, e armazenadas em caixas de papelão [Tedesco et al. 1995], [Bernardi et al. 2014].

A aquisição dos espectros foi realizada diretamente sobre a superfície das amostras, com auxílio de um espectrômetro com fibra óptica. O sistema atuou com uma resolução de 3,2 nm na região de 951 a $2.450 \mathrm{~nm}$, área de varredura de $4 \mathrm{~cm}^{2}$ (regulável), com velocidade de 6 mostras por minuto, conforme orientações do fabricante. Foram adquiridos 5 espectros de cada amostra, e utilizado o espectro médio para representar cada amostra nos modelos de calibração e predição. A Figura 5 apresenta as alterações no perfil da espectroscopia molecular na região do infravermelho próximo das 355 amostras utilizadas nessa fase de modelagem.

\section{Resultados e discussões}

Para gerar o modelo de calibração foram utilizados os dados de infravermelho dos históricos de contextos, um para matéria orgânica e outro para argila. Todas as amostras empregadas foram reanalisadas pelo método oficial (ROLAS) para que pudessem ser feitas as correlações dos dados via algoritmo Partial Least Squares. O mesmo foi configurado para que usasse 18 componentes ou variáveis latentes. Os gráficos da Figura 6 demonstram as performances desses modelos, da matéria orgânica (A), cujo coeficiente de determinação $\left(\mathrm{R}^{2}\right)$ foi de 0.9102 , erro médio de calibração (RMSE) de $0.49 \%$ e erro médio de validação cruzada (RMSECV) de $0.82 \%$, e da argila (B), com $\mathrm{R}^{2}$ de 0.9242 , RMSE de $4.19 \%$ e RMSECV de $8.43 \%$. 


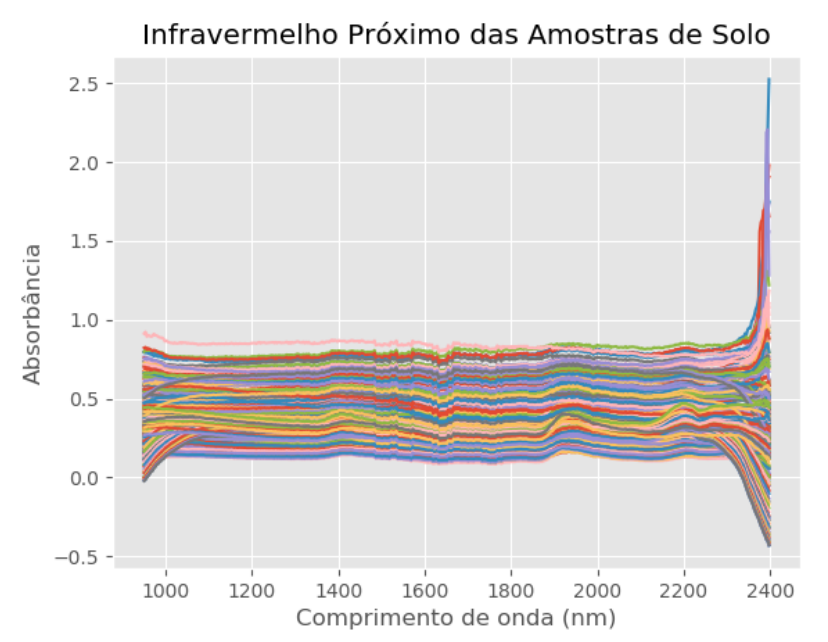

Figura 5. Dados espectrais das amostras de solo
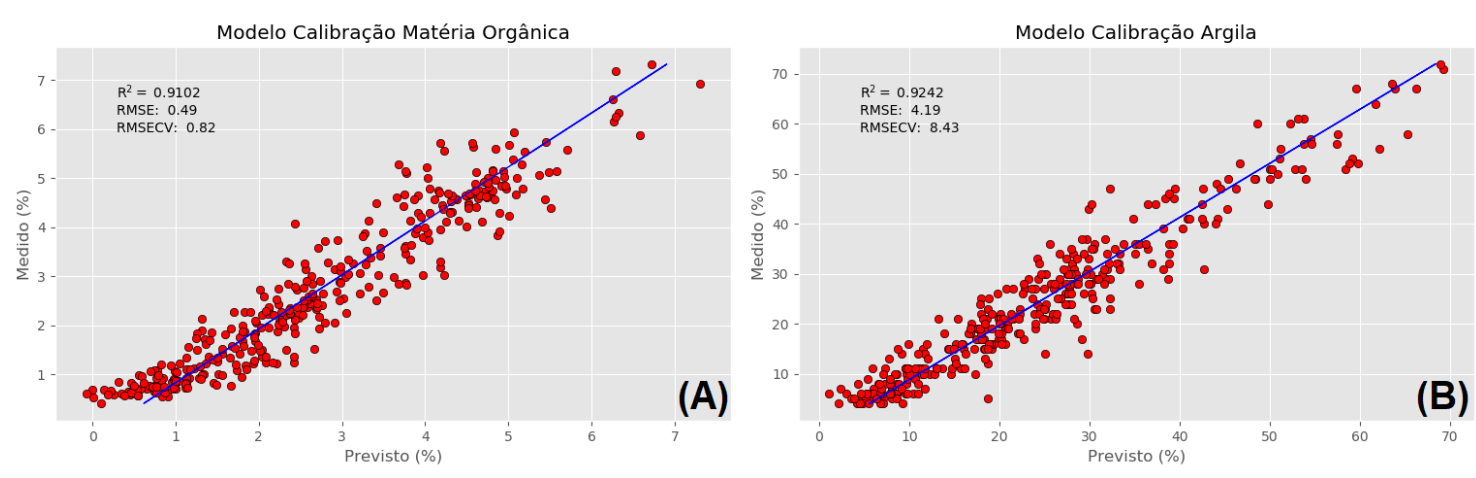

Figura 6. Modelo de calibração para Matéria Orgânica (A) e Argila (B)

Comparando os resultados do modelo de calibração gerados pelos históricos de contextos do Tellus com trabalhos de diferentes autores, foram conseguidos melhores índices de avaliação preditiva. Para matéria orgânica, [Nawar and Mouazen 2019] apresentaram coeficiente de determinação $\left(\mathrm{R}^{2}\right)$ de até 0.84 , porém um RMSE menor, de $0.14 \%$, assim como para argila, [Wetterlind et al. 2015] obtiveram um $\mathrm{R}^{2}$ de 0.76 e um RMSECV de 6.4\%. Os erros RMSE e REMSEC menores desses autores se devem à baixa representatividade amostral. Ambos focaram seus experimentos em lotes de aproximadamente $0.5 \mathrm{Km}^{2}$ enquanto os históricos de contexto do Tellus representaram diversos pontos do Vale do Rio Pardo - RS. Além disso, outros fatores como sensibilidade, reprodutibilidade e interferências dos equipamentos, intrínsecos ao método, poderiam ser também discutidos. Vale ressaltar que o modelo tende melhorar sua capacidade de predição à medida que novos dados de contexto vão encorpando a base histórica.

\section{Conclusão}

Este artigo propôs um modelo computacional denominado Tellus para predição da fertilidade do solo através de históricos de contextos. Com relação a estes, dados de infravermelho foram extraídos para que concentrações de matéria orgânica e argila fossem previstas. Deste modo, foram destacadas as seguintes estratégias de trabalho: predição 
de contextos baseada em modelos de regressão linear, validação dos resultados obtidos e desenvolvimento da arquitetura. Os autores reconhecem que, para predição de argila, apesar de um coeficiente de determinação melhor, os erros de calibração poderiam ser ainda menores. Quanto aos trabalhos futuros, visando melhoria da capacidade preditiva, sugere-se o emprego de técnicas de seleção de regiões espectrais utilizando modelos de similaridade e processamento paralelo, assim como, um estudo mais aprofundado para eliminação de outliers.

\section{Agradecimentos}

Os autores agradecem à Fundação de Amparo à Pesquisa do Estado do Rio Grande do Sul (FAPERGS), à Coordenação de Aperfeiçoamento de Pessoal de Nível Superior - Brasil (CAPES) - Código de Financiamento 001, ao Conselho Nacional de Desenvolvimento Científico e Tecnológico (CNPq), à Universidade de Santa Cruz do Sul e o setor Central Analítica, pelo fornecimento das amostras de solos, e à Universidade do Vale do Rio dos Sinos (Unisinos) pelo apoio ao desenvolvimento desse trabalho. Os autores reconhecem especialmente o apoio do Programa de Pós-Graduação em Computação Aplicada (PPGCA) e do Laboratório de Computação Móvel (Mobilab) da Unisinos.

\section{Referências}

Anzanello, M. J. (2013). Seleção de variáveis para classificação de bateladas produtivas com base em múltiplos critérios. Production, 23(4):858-865.

Barbosa, J., Tavares, J., Cardoso, I., Alves, B., and Martini, B. (2018). Trailcare: An indoor and outdoor context-aware system to assist wheelchair users. International Journal of Human-Computer Studies, 116:1 - 14.

Bernardi, A., Naime, J., Resende, A., Inamasu, R., and Bassoi, L. (2014). Agricultura de precisão: resultados de um novo olhar. Manual de métodos de análise de solos. EMBRAPA, São Carlos - SP, 2 edition.

Blank, S., Bartolein, C., Meyer, A., Ostermeier, R., and Rostanin, O. (2013). IGreen: A ubiquitous dynamic network to enable manufacturer independent data exchange in future precision farming. Computers and Electronics in Agriculture, 98:109-116.

Concepcion, A. R., Stefanelli, R., and Trinchero, D. (2014). A wireless sensor network platform optimized for assisted sustainable agriculture. In IEEE Global Humanitarian Technology Conference (GHTC 2014), pages 159-165. IEEE.

Costa, N. R., Carvalho, M. d. P. e., Dal Bem, E. A., Dalchiavon, F. C., and Caldas, R. R. (2014). Produtividade de laranja correlacionada com atributos químicos do solo visando a zonas específicas de manejo. Pesquisa Agropecuária Tropical, 44(4):391398.

Dey, A. K., Abowd, G. D., and Salber, D. (2001). A Conceptual Framework and a Toolkit for Supporting the Rapid Prototyping of Context-Aware Applications. $\mathrm{Hu}$ man-Computer Interaction, 16(2-4):97-166.

Ferreira, M. M. C. (2015). Quimiometria: conceitos, métodos e aplicações. Editora da Unicamp, Campinas-SP. 
Goap, A., Sharma, D., Shukla, A., and Rama Krishna, C. (2018). An iot based smart irrigation management system using machine learning and open source technologies. Computers and Electronics in Agriculture, 155:41-49.

Griebeler, G., da Silva, L. S., Cargnelutti Filho, A., and Santos, L. d. S. (2016). Avaliação de um programa interlaboratorial de controle de qualidade de resultados de análise de solo. Revista Ceres, 63(3):371-379.

Huong, T. T., Thanh, N. H., Van, N. T., Dat, N. T., Long, N. V., and Marshall, A. J. (2018). Water and energy-efficient irrigation based on markov decision model for precision agriculture. 2018 IEEE Seventh International Conference on Communications and Electronics (ICCE), pages 51-56.

Muñoz, J. D. and Kravchenko, A. (2011). Soil carbon mapping using on-the-go near infrared spectroscopy, topography and aerial photographs. Geoderma, 166(1):102110.

Nawar, S. and Mouazen, A. (2019). On-line vis-nir spectroscopy prediction of soil organic carbon using machine learning. Soil and Tillage Research, 190:120 - 127.

Rosa, J. H., Barbosa, J. L. V., Kich, M., and Brito, L. (2015). A Multi-Temporal Contextaware System for Competences Management. International Journal of Artificial Intelligence in Education, 25(4):455-492.

Santos, U. J. L., da Rosa Righi, R., and da Costa, C. A. (2018). Compreendendo o desempenho de gerenciadores de contexto para internet das coisas. In $10^{\circ}$ Simpósio Brasileiro de Computação Ubíqua e Pervasiva (SBCUP), volume 10, Porto Alegre, RS, Brasil. SBC.

SAP AG (2007). Standardized technical architectur e modeling - conceptual and design level. http://www. fmc-modeling.org/download/fmc-and-tam/ SAP-TAM_Standard.pdf Accessed 23-Jan-2019.

Schmitz, M., Martini, D., Kunisch, M., and Mösinger, H.-J. (2009). agroXML Enabling Standardized, Platform-Independent Internet Data Exchange in Farm Management Information Systems. In Metadata and Semantics, pages 463-468. Springer US, Boston, MA.

Tedesco, M., Gianello, C., Bissani, C., Bohnen, H., and Volkweiss, S. (1995). Análise de solo, plantas e outros materiais. UFRGS/Departamento de Solos, Porto Alegre, 2 edition.

Treboux, J. and Genoud, D. (2018). Improved machine learning methodology for high precision agriculture. 2018 Global Internet of Things Summit (GIoTS), pages 1-6.

Wetterlind, J., Piikki, K., Stenberg, B., and Söderström, M. (2015). Exploring the predictability of soil texture and organic matter content with a commercial integrated soil profiling tool. European Journal of Soil Science, 66(4):631-638. 